\title{
Egg transfer in the Mongolian gerbil (Meriones unguiculatus) during lactational delay of implantation
}

\author{
M. L. Norris and W. F. Rall \\ A.R.C. Institute of Animal Physiology, Animal Research Station, 307 Huntingdon Road, \\ Cambridge CB3 OJQ, U.K.
}

\begin{abstract}
Summary. Lactating pregnant gerbils in which one oviduct was ligated during early pregnancy were used on Day 6-14 p.c. as recipients for eggs (morulae and blastocysts) recovered on Day 6-12 p.c. from lactating donors. Eggs were transferred to the ligated (experimental) horn and the litter was removed. About half of the recipients had implantations in the non-ligated (control) horn 15 days after litter removal. Of these 40 females, 37 had implantations in the experimental horn and 32 had some normal fetuses present. Of 179 eggs transferred into the ligated uteri, $61 \%$ implanted and $41 \%$ developed into normal fetuses. The time after copulation of recipient and donor did not appear to influence the number of implantations or post-implantation development of the transferred eggs.
\end{abstract}

\section{Introduction}

Since its introduction as a new laboratory rodent (Schwentker, 1963; Rich, 1968), the Mongolian gerbil (Meriones unguiculatus) is the only member of the sub-family Gerbillinae now widely used for experimental purposes. The recent availability of coat colour variants has greatly facilitated the usefulness of this animal for investigations of basic questions of reproductive physiology.

To date there is only one preliminary report concerned with the transfer of gerbil eggs; pseudopregnant mice were used as recipients for blastocysts cultured in vitro from the 4- to 8-cell stage (Fisher \& Fischer, 1973). The production of pseudopregnant gerbil recipients is complicated, mainly because of the difficulties associated with their behaviour under laboratory conditions. Mature gerbils are normally maintained monogamously, and replacing the male of an established pair with a vasectomized male can lead to extreme aggression by adult females. Also, unlike the mouse, the copulatory plug in the gerbil is small, very deep seated in the vagina, and disappears relatively quickly (Norris \& Adams, 1981a). Attempts to induce pseudopregnancy in the gerbil by cervical stimulation have yielded disappointing results compared with those obtained using the same technique in rats (Wu, 1974).

However, some aspects of the reproductive behaviour of the gerbil are particularly amenable to experimental manipulation. About $80 \%$ of gerbils mate within $24 \mathrm{~h}$ after parturition, with a well defined peak of activity occurring between $16: 00$ and $20: 00 \mathrm{~h}$ on the day of parturition (Norris \& Adams, 1981a). Implantation normally occurs on Day 8, but when 5-6 young are being suckled, implantation may be delayed up to Day 26 (Norris \& Adams, 1981b). Implantation may be readily induced at any stage during the delay period by removing the litter (Norris, 1979). The purpose of the present study was to examine the use of this extended delay period for assessing the viability of eggs after transfer and litter removal. 


\section{Materials and Methods}

All animals came from the colony at the Animal Research Station, the management of which was as described by Norris \& Adams (1981b). A total of 157 mature females were kept as monogamous pairs from 2 months of age, and used at 3-10 months of age following the birth of at least 1 litter. Routine procedures, such as inspections for litters and vaginal lavage, were carried out daily at $09: 00-10: 00 \mathrm{~h}$. New litters were adjusted to 5-6 young, and this number was maintained by crossfostering whenever necessary. On the morning after parturition, mating was confirmed by the presence of spermatozoa in the vaginal smear (= Day 1 post coitum (p.c.)); mated females were allocated to either a donor $(\mathrm{N}=76)$ or recipient $(\mathrm{N}=81)$ group.

\section{Donor animals}

Donors were killed on Day 6-14, and the ovaries, oviducts and uteri were removed and carefully dissected. The corpora lutea were counted, and the oviducts of Day-6 donors were flushed from the ampullary end with approximately $0.25 \mathrm{ml}$ sterile PBI solution (Whittingham, 1974) into a punted watch glass. The uterine horns of all donors were flushed with approximately $1 \mathrm{ml} \mathrm{PB} 1$ solution $(0.5$ $\mathrm{ml}$ in either direction). Oviducal and uterine flushings were immediately searched using a stereomicroscope, eggs were counted and morphologically assessed. Spherical morulae and cavitated early blastocysts were usually recovered from the oviducts of Day-6 donors. Fully cavitated, spherical blastocysts were recovered on Day 7, whereas blastocysts recovered from Day-8-14 donors were usually elliptical. Eggs of normal appearance were washed with fresh PB1 solution and transferred within $20 \mathrm{~min}$ of recovery.

\section{Recipient animals}

On Day 2-5, when all of the eggs are still in the oviducts, recipient animals were anaesthetized by inhalation of methoxyflurane (Norris \& Miles, 1982), and a small flank incision was made on one side to expose the ovary and utero-tubal junction. After confirming the presence of corpora lutea, a ligature (Armofil No. 1.5 suture material: Armour Pharmaceutical Co. Ltd, Eastbourne, U.K.) was placed at the utero-tubal junction. The abdominal wall and skin layer were closed separately with sutures.

At the time of egg transfer (Day 6-14), recipients were anaesthetized as above and the ligated uterine horn was exposed through the original incision. The uterus was punctured just below the ligature with a size 10 embroidery needle (Milward \& Sons, Studley, Warwickshire) and the eggs were transferred in about $2 \mu \mathrm{l}$ medium PB1 into the uterine lumen by using a mouth-operated micropipette. The uterine horn was gently returned into the abdominal cavity, and the incision resutured. After recovery from anaesthesia, recipients were returned to their original cages. The young were removed from the recipients at the time of egg transfer, except for one group of 4 Day- 6 recipients. At this stage the native eggs are still in the oviducts, and we thought that a $24-\mathrm{h}$ delay in removal of the young might be necessary for synchronous implantation in the control and experimental uteri.

\section{Implantation and fetal development}

Recipients were autopsied 15 days after removal of the young. The patency of the utero-tubal duct at the ligature was checked and the ovaries, oviducts and uteri were removed and dissected. The corpora lutea were counted, and the uteri opened and examined for normal fetuses/placentae and regressing implantations (moles). Fetuses, placentae and moles were gently eased away from the maternal tissue, blotted and weighed. Fetal crown-rump lengths were measured using dial calipers (Mitutoya, Tokyo, Japan). 


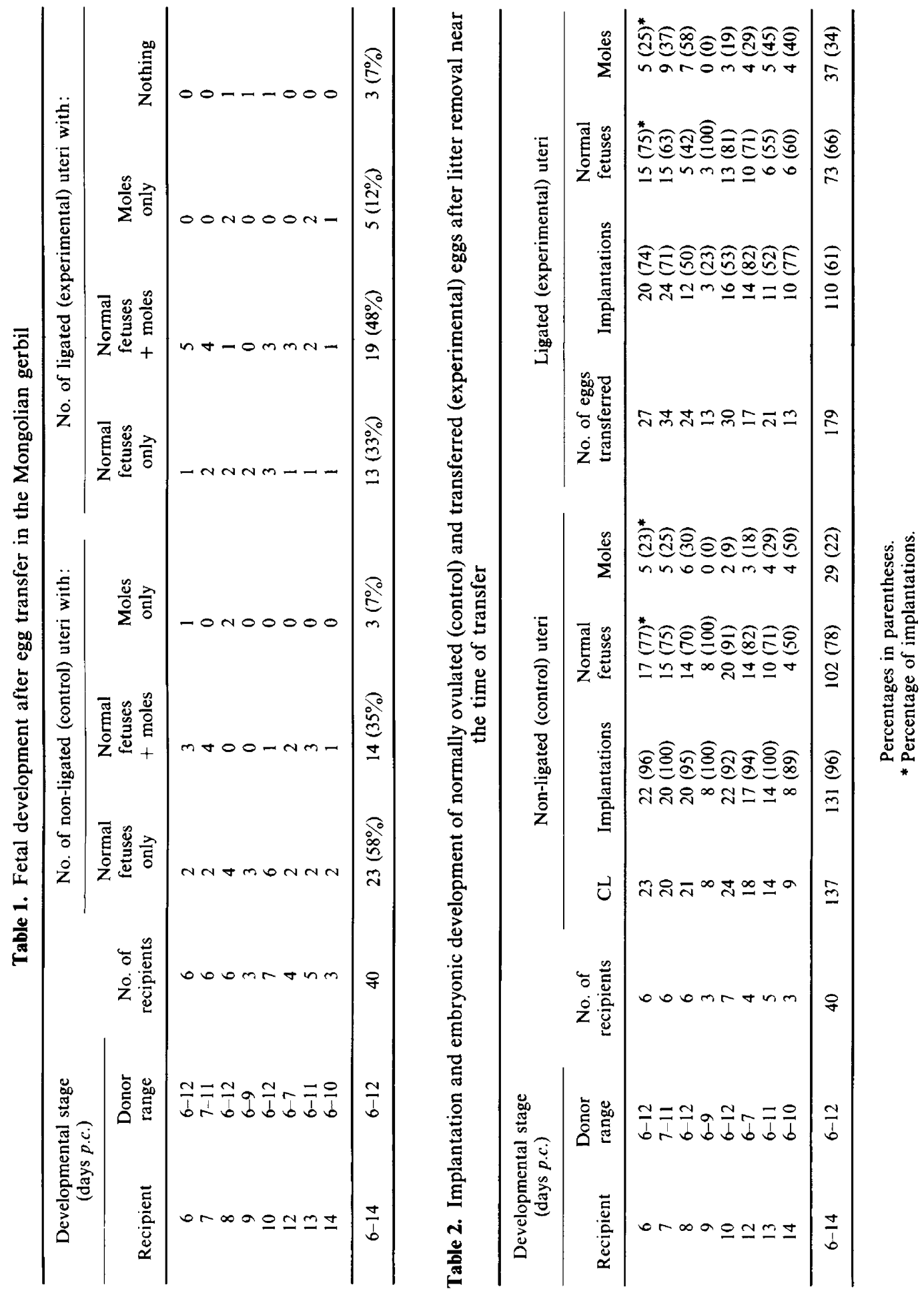




\section{Results}

Of the 81 recipients autopsied, $40(49 \%)$ had implantations present in the non-ligated (control) horn, and $41(51 \%)$ were without implantations in either horn. For unilateral uterine transfers made on Day 6, implantations were present in both horns in 3 out of 6 females who had had the young removed at the time of transfer, and in 3 out of 4 females for which the litter was removed $24 \mathrm{~h}$ after transfer.

\section{Females without implantations}

In these 41 recipients an average of 5.1 (3-8) eggs were transferred into the ligated horns on Day 6-14. The mean age (p.c.) of the recipients and donors at the time of transfer was 9.9 (6-14) days and $8 \cdot 1(6-14)$ days respectively. No corpora lutea were found in the ovaries of $32(78 \%)$ of these females at autopsy.

\section{Females with implantations in the control horn}

In these 40 recipients an average of $4.5(3-7)$ eggs were transferred into the ligated horns on Day 6-14. The mean age (p.c.) of the recipients and donors at the time of transfer was $9 \cdot 5(6-14)$ days and $8 \cdot 1(6-12)$ days respectively. Most of these recipients (37/40) had implantations in the ligated (experimental) horn (Table 1). Most $(96 \%)$ of the corpora lutea were represented by implantations in the control horns, whereas $61 \%$ of the transferred eggs implanted in the experimental horns (Table 2). The experimental horns had a lower proportion of the implantations present as normal fetuses, and a greater proportion as moles, than did the control horns. When the relative age (p.c.) of recipient uteri and transferred eggs was compared (Table 3 ) there was no obvious difference in the implantation rate or fetal development. There were no significant differences between the fetal, placental and mole values for the two horns (Table 4), suggesting a similar time of implantation and rate of post-implantation development.

Table 3. The effect of stage (p.c.) of donor and recipient on the development of transferred gerbil eggs

\begin{tabular}{lccccc}
\hline & \multicolumn{4}{c}{ Number (\%) } \\
\cline { 2 - 6 } $\begin{array}{c}\text { Stage of donors } \\
\text { relative to recipients }\end{array}$ & Transfers & $\begin{array}{c}\text { Recipients with } \\
\text { normal fetuses } \\
\text { in the ligated horn }\end{array}$ & $\begin{array}{c}\text { Eggs } \\
\text { transferred }\end{array}$ & Implantations & $\begin{array}{c}\text { Normal } \\
\text { fetuses }\end{array}$ \\
\hline Younger (1-8 days) & 21 & $17(81)$ & 88 & $54(61)$ & $38(43)$ \\
Identical & 10 & $7(70)$ & 46 & $27(59)$ & $15(33)$ \\
Older (2-6 days) & 9 & $8(89)$ & 45 & $29(64)$ & $20(44)$ \\
\hline
\end{tabular}

Table 4. Embryonic development in post-parturient, pregnant recipient gerbils $(\mathrm{N}=24)$ with fetuses present in both uterine horns 15 days after litter removal

\begin{tabular}{|c|c|c|c|c|c|c|}
\hline \multirow[b]{3}{*}{ Uterine horns } & \multicolumn{4}{|c|}{ Normal fetuses } & \multirow{2}{*}{\multicolumn{2}{|c|}{ Moles }} \\
\hline & \multirow[b]{2}{*}{ No. } & \multirow{2}{*}{$\begin{array}{l}\text { Crown-rump } \\
\text { length }(\mathrm{mm})\end{array}$} & \multicolumn{2}{|c|}{ Weight (mg) } & & \\
\hline & & & Fetus & Placenta & No. & Wt (mg) \\
\hline Non-ligated (control) & 60 & $17 \cdot 1 \pm 0 \cdot 2$ & $233.2 \pm 4.7$ & $579.7 \pm 15 \cdot 3$ & 13 & $57 \cdot 2 \pm 12 \cdot 5$ \\
\hline Ligated (experimental) & 55 & $17.0 \pm 0.2$ & $233.7 \pm 5.3$ & $557.0 \pm 13.9$ & 15 & $59.7 \pm 10.6$ \\
\hline
\end{tabular}




\section{Discussion}

Our results show that $41 \%$ of the transferred eggs develop into normal fetuses in recipients that maintain pregnancy. This result compares favourably with an earlier report by Mantalenakis $\&$ Ketchel (1967) on the development of Day-5 rat blastocysts transferred to post-parturient, lactating recipients on Day 5-13 of pseudopregnancy $(51 \%)$. However, when compared to egg transfer in the mouse, the proportion of transferred gerbil eggs developing into normal fetuses is low. For example, Whittingham, Lyon \& Glenister (1977; Table 3) found that $67 \%$ of mouse embryos transferred to pseudopregnant recipients developed into normal fetuses. However, of the total number of implantations recorded from transferred eggs, a similar level of post-implantation death was found in the gerbil $(34 \%)$ and mouse $(27 \%$, Whittingham et al., 1977).

Ovariectomy and progesterone therapy offers similar physiological conditions to those in the present study. Under these conditions, implantation has been successfully induced by exogenous oestrogen at or after the transfer of eggs in the rat (Psychoyos, 1961) and mouse (Humphrey, 1967). However, Humphrey (1969) has noted that the success of implantation depends on the day of transfer relative to the start of progesterone therapy.

Removal of young from many post-parturient rodents is thought to result in increased ovarian activity, followed by increased oestrogenic activity in the uterus (Zeilmaker, 1964; McLaren, 1968; Bindon, 1970; Norris, 1979). Our results provide three lines of evidence on the timing of implantation-related events in the gerbil. (1) The unilateral transfer of eggs to Day-6 recipients provides mature blastocysts in the experimental horn, while the native eggs are still cavitating in the contralateral oviduct. Successful implantation of these native eggs, irrespective of whether the litter was removed at the time of transfer or $24 \mathrm{~h}$ later, means that the interval between litter removal and the endogenous stimulus to implantation is sufficiently long to permit normal preimplantation events (e.g. blastocyst maturation and uterine spacing). (2) The autopsy results (Table 4) suggest that implantation was induced simultaneously in both horns irrespective of the relative stage post coitum of the donor eggs and recipient. (3) Fetal measurements are identical with those obtained earlier for Day-20 fetuses from non-lactating, post-parturient gerbils (Bagwell \& Leavitt, 1974). Day 20 is equivalent to 12 days after implantation, which indicates that implantation must have occurred within 3 days of litter removal.

Failure of half the recipients to maintain pregnancy does not appear to be related to the ages ( p.c.) of either the donor or recipient. However, this failure is probably correlated with the absence of corpora lutea at autopsy. Luteal failure may result from the physiological trauma of two surgical operations. This suggestion can be tested by eliminating either one operation by transferring genetically marked eggs (e.g. albino or black coat variants) or both operations by non-surgical transfer (Moler, Donahue \& Anderson, 1979). However, studies on the mouse (McLaren \& Michie, $1956,1959)$ indicate that some competitive interaction between native and transferred gerbil eggs might be expected, especially when native blastocysts have already become spaced in the uterine horn before transfer.

In conclusion, the lactating recipients used in the present study offer several advantages: a 9day period during which transfer of morulae or blastocysts can be made; native eggs in the nonligated horn act as controls; and implantation can be induced bilaterally by litter removal.

W.F.R. is supported by the Stanley Thomas Johnson Foundation. We thank Dr C. Polge and Dr C. E. Adams for helpful comments during the preparation of the manuscript.

\section{References}

Bagwell, J.N. \& Leavitt, W.W. (1974) Prenatal size-age relationship and external morphology in the Mongolian gerbil (Meriones unguiculatus). Am. J. Anat. 140, $117-128$.

Bindon, B.M. (1970) Preimplantation changes after litter removal from suckling mice. $J$. Endocr. 46, 511-516.
Fisher, D.L. \& Fischer, T.V. (1973) Recovery and in vitro culture of preimplantation Mongolian gerbil embryos. Teratology 7, A14-A15, Abstr.

Humphrey, K.W. (1967) The development of viable embryos after ovum transfers to long term ovariectomized mice. Steroids 9, 53-56. 
Humphrey, K.W. (1969) Induction of implantation of blastocysts transferred to ovariectomized mice. $J$. Endocr. 44, 299-305.

Mantalenakis, S.J. \& Ketchel, M.M. (1967) Blastocyst implantation during post-partum pseudopregnancy in the rat. $J$. Endocr. 37, 385-391.

McLaren, A. (1968) A study of blastocysts during delay and subsequent implantation in lactating mice. $J$. Endocr. 42, 453-463.

McLaren, A. \& Michie, D. (1956) Studies on the transfer of fertilized mouse eggs to uterine foster mothers. I. Factors affecting the implantation and survival of native and transferred eggs. J. exp. Biol. 33, 394-416.

McLaren, A. \& Michie, D. (1959) Studies on the transfer of fertilized mouse eggs to uterine foster mothers. II. The effect of transferring large numbers of eggs. $J$. exp. Biol. 36, 40-50.

Moler, T.L., Donahue, S.E. \& Anderson, G.B. (1979) A simple technique for non-surgical embryo transfer in mice. Lab. Anim. Sci. 29, 353-356.

Norris, M.L. (1979) Studies on reproduction in the female Mongolian gerbil, Meriones unguiculatus, with particular reference to ovum implantation. Ph.D. thesis, C.N.A.A.

Norris, M.L. \& Adams, C.E. (1981a) Time of mating and associated changes in the vaginal smear of the postparturient Mongolian gerbil (Meriones unguiculatus). Lab. Anim. 15, 193-198.
Norris, M.L. \& Adams, C.E. (1981b) Mating post partum and length of gestation in the Mongolian gerbil (Meriones unguiculatus). Lab. Anim. 15, 189-191.

Norris, M.L. \& Miles, P. (1982) An improved, portable machine designed to induce and maintain surgical anaesthesia in small laboratory rodents. Lab. Anim. 16, 227-230.

Psychoyos, M.A. (1961) Nouvelles recherches sur l'ovoimplantation. C. r. hebd. Séanc. Acad. Sci., Paris D 252, 2306-2307.

Rich, S.T. (1968) The Mongolian gerbil (Meriones unguiculatus) in research. Lab. Anim. Care 18, 235243.

Schwentker, V. (1963) The gerbil. A new laboratory animal. Illinois Vet. 6, 5-9.

Whittingham, D.G. (1974) Embryo banks in the future of developmental genetics. Genetics, Princeton 78, 395402.

Whittingham, D.G., Lyon, M.F. \& Glenister, P.H. (1977) Long-term storage of mouse embryos at $-196^{\circ} \mathrm{C}$ : the effect of background radiation. Genet. Res. 29, 171181.

Wu, J.T. (1974) Artificial insemination and induction of pregnancy in the Mongolian gerbil (Meriones unguiculatus). J. Reprod. Fert. 37, 139-140.

Zeilmaker, G.H. (1964) Quantitative studies on the effect of the suckling stimulus on blastocyst implantation in the rat. Acta endocr., Copenh. 46, 483-492.

Received 31 August 1982 